EPJ Web of Conferences 19, 02002 (2012)

DOI: $10.1051 /$ epjconf/20121902002

(C) Owned by the authors, published by EDP Sciences, 2012

\title{
Mapping the Galactic halo with main-sequence and RR Lyrae stars
}

\section{B. Sesar ${ }^{\mathrm{a}}$}

\author{
Division of Physics, Mathematics and Astronomy, California Institute of Technology, \\ Pasadena, CA 91125, USA
}

\begin{abstract}
We present an analysis of Galactic halo structure, substructure, and metallicity traced by mainsequence and RR Lyrae stars selected from the SDSS stripe 82 and CFHT Legacy Survey data sets. The main result of the study based on SDSS stripe 82 data is a 2D map of the Galactic halo that reaches distances of $100 \mathrm{kpc}$ and traces previously known and new halo substructures, such as the Sagittarius and Pisces tidal streams. We present strong direct evidence, based on both RR Lyrae and main-sequence stars, that the halo stellar number density profile significantly steepens beyond $30 \mathrm{kpc}$ from the Galactic center. The steepening of the density profile beyond $30 \mathrm{kpc}$ is also evident in the distribution of main-sequence stars observed by the CFHT Legacy Survey along four Galactic lines of sight. In the two CFHT sightlines where we do not detect significant substructure, the median metallicity is found to be independent of distance within systematic uncertainties $([\mathrm{Fe} / \mathrm{H}] \sim-1.5 \pm 0.1 \mathrm{dex}$ within $30 \mathrm{kpc}$ of the Galactic Center).
\end{abstract}

\section{GALACTIC HALO AS THE ROSETTA STONE FOR GALAXY FORMATION}

Studies of the Galactic halo can help constrain the formation history of the Milky Way and the galaxy formation process in general. For example, state-of-the-art simulations of galaxy formation predict numerous substructures, such as tidal tails and streams, in halos of Milky Way-size galaxies (e.g., see Fig. 14 in [12] or Fig. 6 in [5]). The amount, morphology, kinematics, and chemical composition of these substructures depend on the accretion history of the simulated galaxy ([12], [5]). Therefore, if we map the substructures in the Galactic halo and compare the resultant maps with simulations, we will be able to constrain the formation history of the Milky Way.

Maps of the Galactic halo that probe galactocentric distances $\left(R_{\mathrm{GC}}\right)$ of $\sim 20-30 \mathrm{kpc}$ have already been made using near turn-off main sequence stars ([13], [1]) selected from the Sloan Digital Sky Survey (SDSS; [20]). Unfortunately, the largest discrepancy between simulated halos with different formation histories occurs at distances beyond $30 \mathrm{kpc}$ (e.g., compare panels in [5] Fig. 6). Therefore, to discriminate between different formation histories we need to map the Galactic halo beyond $30 \mathrm{kpc}$ and to do that we need to use tracers that are brighter than main-sequence turnoff stars.

\section{MAPPING THE GALACTIC HALO WITH SDSS STRIPE 82 RR LYRAE STARS}

To map the Galactic halo beyond $30 \mathrm{kpc}$, we used $\sim 400$ type- $a b$ RR Lyrae stars selected from the SDSS stripe 82 region (for more details on SDSS stripe 82 and the selection algorithm see [16]). The 2D (right

ae-mail: bsesar@astro.caltech.edu

This is an Open Access article distributed under the terms of the Creative Commons Attribution-Noncommercial License 3.0, which permits unrestricted use, distribution, and reproduction in any noncommercial medium, provided the original work is properly cited. 


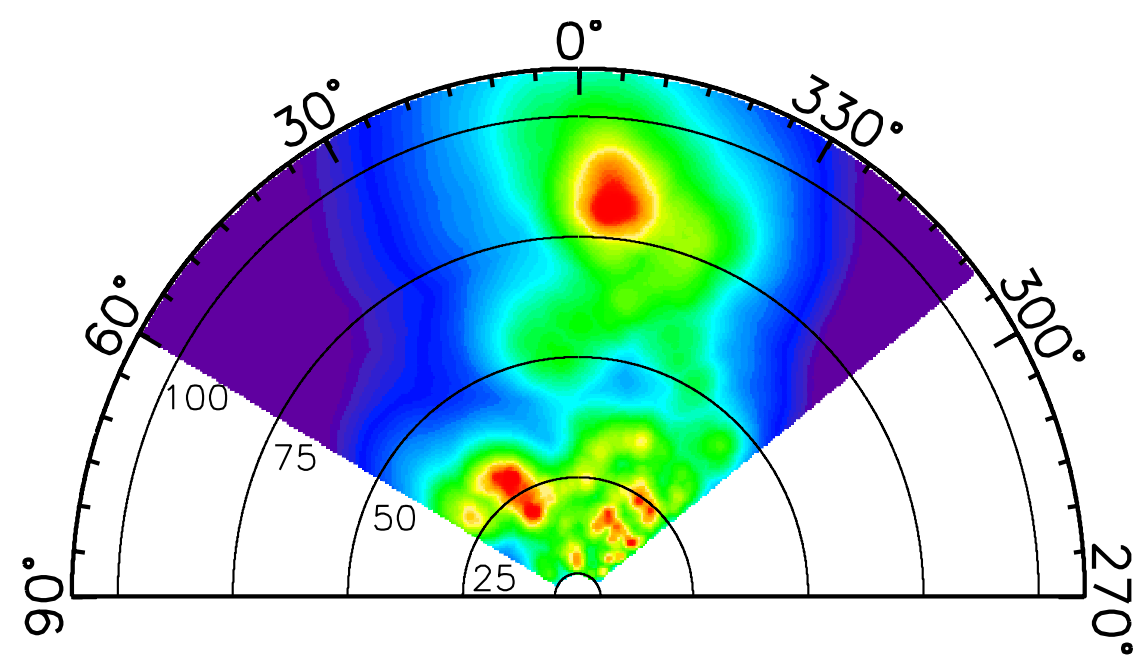

Figure 1. Halo substructures traced by RR Lyrae stars in SDSS stripe 82. The heliocentric distance in kpc is in the radial direction and angle indicates equatorial right ascension. The red color indicates substructures, green color shows the smooth halo, and the blue color shows regions where there are almost no stars. The Pisces Overdensity/Stream is located $\sim 80 \mathrm{kpc}$ from the Sun at R.A. $\sim 355 \mathrm{deg}$, the trailing arm of the Sagittarius tidal stream [9] is passing through the stripe 82 plane at (R.A., helio.dist. $)=(\sim 30 \mathrm{deg}, 25 \mathrm{kpc})$, and the Hercules-Aquila Cloud is located at $(\sim 330 \mathrm{deg},<25 \mathrm{kpc})$. Figure adapted from [16] Fig. 11.

ascension and heliocentric distance) number density distribution of RR Lyrae stars was obtained by applying a Bayesian number density estimator [10] on the spatial distribution of RR Lyrae stars. The calculated number densities of RR Lyrae stars were compared to values predicted by a smooth, oblate halo model with a density power-law slope of -2.7 (best-fit halo model from [13]).

Figure 1 shows the comparison of observed and model-predicted number densities on a logarithmic scale. The green regions are in agreement with the smooth halo model, red regions are overdense by a factor of 10 , and blue regions are underdense by a factor of 10 compared to the model. Within about $30 \mathrm{kpc}$, the observed halo follows the oblate power-law model. Two substructures are clearly visible: the Sgr trailing arm and the Hercules-Aquila Cloud [2]. Beyond $30 \mathrm{kpc}$, the model predicts more stars than what is actually observed, indicating that the observed number density profile steepens.

The overdensity seen in Fig. 1 at about $80 \mathrm{kpc}$ was first reported in [15] ("J" clump) and was later renamed the Pisces overdensity by [19]. [14] and [17] have observed this substructure spectroscopically and have detected two velocity peaks, suggesting that the substructure may be a tidally disrupted dwarf galaxy.

\section{MAPPING THE GALACTIC HALO WITH CFHT MAIN-SEQUENCE STARS}

The steepening of the halo stellar number density profile beyond $30 \mathrm{kpc}$ is also present when mainsequence stars are used as tracers, as evident in deep coadded SDSS data (see Fig. 24 in [16]) and CFHT Legacy Survey data (CFHTLS, see Fig. 2 below).

We find that the halo stellar number density profile becomes steeper at Galactocentric distances greater than $R_{\text {gal }} \sim 28 \mathrm{kpc}$, with the power law index changing from $n_{\text {inner }}=-2.62 \pm 0.04$ to $n_{\text {outer }}=$ $-3.8 \pm 0.1$. We measure the oblateness of the halo to be $q \equiv c / a=0.70 \pm 0.01$ and detect no evidence of it changing across the range of probed distances $\left(5<R_{\text {gal }}<30 \mathrm{kpc}\right)$. The mathematical 


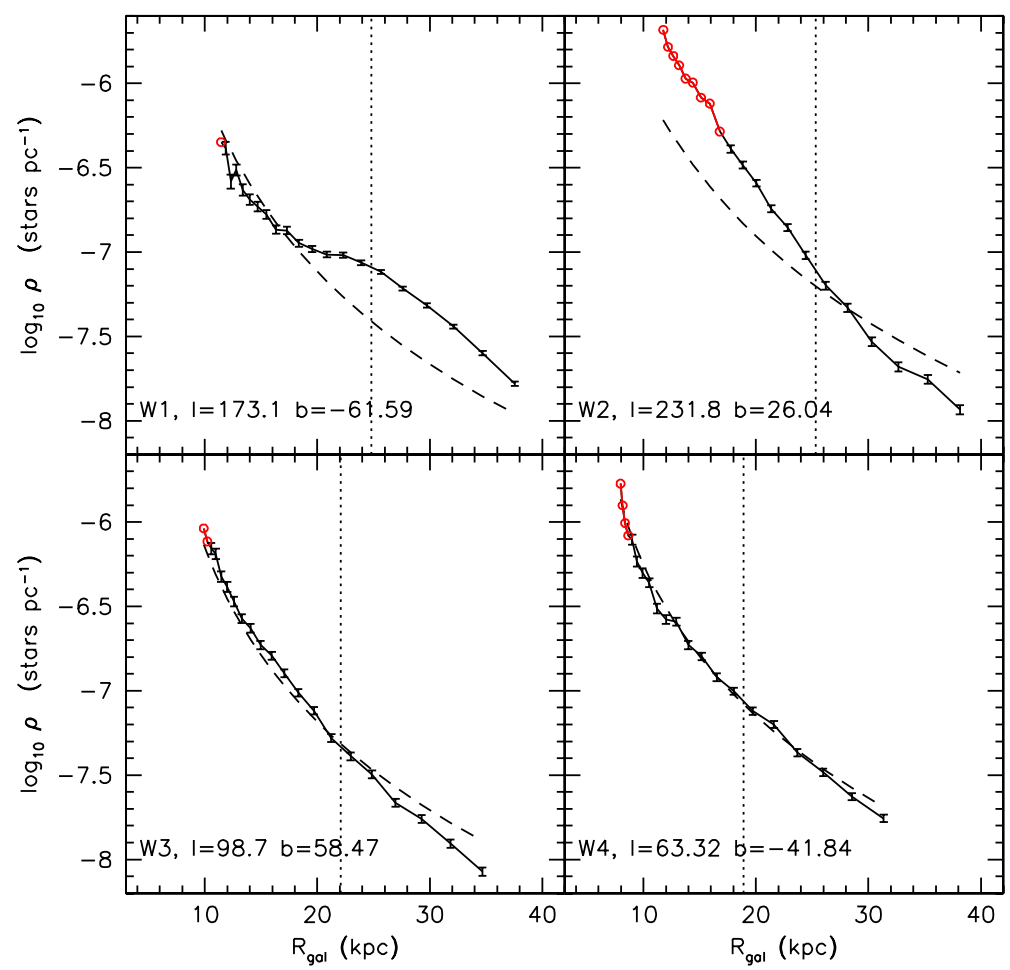

Figure 2. Stellar number density, measured in four CFHTLS wide-area survey beams as a function of distance from the Galactic center, $R_{\text {gal }}$. Open circles denote the measurements within $5 \mathrm{kpc}$ of the Galactic plane, where the contamination by disk stars may be greater than $10 \%$. For clarity, the symbols have been connected by solid lines. Overplotted as a dashed line is the oblate power law halo model from . Its overall normalization has been adjusted to fit the W3 and W4 data at $R_{\text {gal }}<25 \mathrm{kpc}$, as well as W1 data points satisfying $R_{\text {gal }}<15 \mathrm{kpc}$ (to avoid contamination by the Sagittarius stream). The vertical line shows the [13] (hereafter J08) distance limit. The excess density at $R_{\text {gal }}>15 \mathrm{kpc}$ in the W1 field can be associated with the Sagittarius stream, while the overdensity at $R_{\text {gal }}<25 \mathrm{kpc}$ in the W2 beam is consistent with the location of the Monoceros stream. Beyond $R_{\text {gal }} \sim 35 \mathrm{kpc}$, the broken power law model (Eq. 1) provides a much better fit to the data than the single power law J08 model (e.g., the J08 model overpredicts the halo stellar number density by $>50 \%$ in W2 and W3 beams at $R_{\text {gal }}>35 \mathrm{kpc}$ ).

representation of our best-fit model is shown in Eq. 1, with $R_{\mathrm{br}} \equiv R_{\mathrm{gal}}$.

$$
\begin{gathered}
R_{e}=\left(x^{2}+y^{2}+\frac{z^{2}}{q}\right)^{\frac{1}{2}} \\
\rho(x, y, z) \propto \begin{cases}\left(R_{e}\right)^{n_{\text {inner }},} & R_{e}<R_{\mathrm{br}} \\
\left(R_{e}\right)^{n_{\text {outer }},} & R_{e}>R_{\mathrm{br}}\end{cases}
\end{gathered}
$$

The availability of $u$-band observations in CFHTLS allows the usage of the [11] photometric metallicity method. We have applied this method to main-sequence stars observed by CFHTLS and have estimated their metallicity on a per-star basis. The median metallicity of main-sequence stars as a function of distance is shown in Fig. 3 below.

We see no evidence of change in halo metallicity within the range of probed distances ( 5 to $35 \mathrm{kpc}$ from the Galactic Center). The halo metallicity ranges between $[\mathrm{Fe} / \mathrm{H}] \sim-1.4 \mathrm{dex}$ and $[\mathrm{Fe} / \mathrm{H}] \sim$ -1.6 dex, and averages at $[\mathrm{Fe} / \mathrm{H}] \sim-1.5 \mathrm{dex}$. This result runs contrary to [4] and [6] studies which 
EPJ Web of Conferences

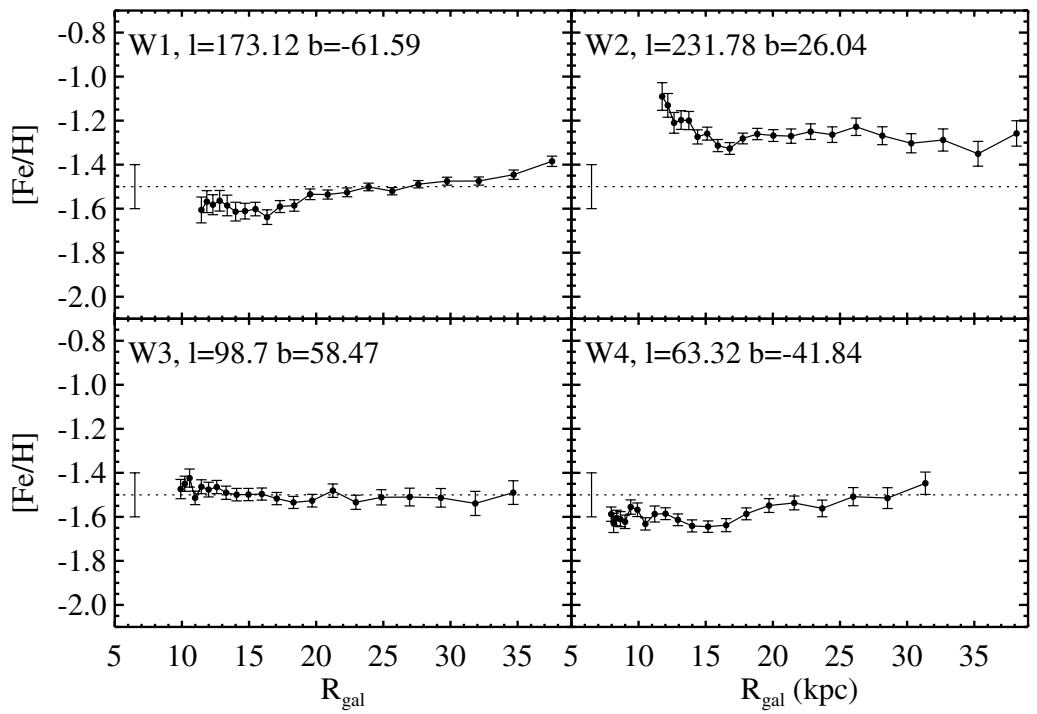

Figure 3. Median photometric metallicity (symbols with error bars) measured in four CFHTLS wide survey beams as a function of distance from the Galactic center, $R_{\text {gal }}$. The error bars show error in the median and the error bar at $(6.5,-1.5)$ shows the systematic uncertainty in the adopted photometric metallicity method $(\sim 0.1 \mathrm{dex},[11])$. Within $R_{\text {gal }} \sim 30 \mathrm{kpc}$, the median metallicity is independent of distance and ranges from $-1.4<[\mathrm{Fe} / \mathrm{H}]<-1.6$. The change in metallicity at $R_{\text {gal }} \sim 15 \mathrm{kpc}$, reported by [4] and [6], is not evident. Apparently higher metallicity in the W2 beam ([Fe/ $\mathrm{H}] \sim-1.3 \mathrm{dex})$ may be due to $u$ band calibration issues (see Section 5 in [18] for a discussion).

report a metallicity of $[\mathrm{Fe} / \mathrm{H}] \sim-1.6$ dex within $R_{\text {gal }} \lesssim 15 \mathrm{kpc}$, and $[\mathrm{Fe} / \mathrm{H}] \sim-2.2$ dex beyond. Only the in situ spectroscopic metallicities of distant main sequence stars may provide a definitive answer to this discrepancy. With the sky density of near-MSTO stars at high Galactic latitudes of about

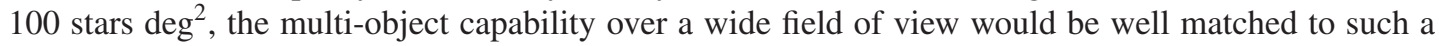
program.

\section{References}

[1] Bell, E. F. et al. 2008, ApJ, 680, 295

[2] Belokurov, V. et al. 2007, ApJ, 657, 89

[3] Bullock, J. S. \& Johnston, K. V. 2005, ApJ, 635, 931

[4] Carollo, D. et al. 2007, Nature, 450, 1020

[5] Cooper, A. P. et al. 2010, MNRAS, 406, 744

[6] de Jong, J. T. A., Yanny, B., Rix, H.-W., Dolphin, A. E., Martin, N. F., \& Beers, T. C. 2010, ApJ, 714,663

[7] De Lucia, G. \& Helmi, A. 2008, MNRAS, 391, 14

[8] Font, A. S. et al. 2008, ApJ, 673, 215

[9] Ivezić, Ž. et al. 2003, Mem. Soc. Astron. Italiana, 74, 978

[10] Ivezić, Ž. et al. 2005, AJ, 129, 1096

[11] Ivezić, Ž. et al. 2008, ApJ, 684, 287

[12] Johnston, K. V. et al. 2008, ApJ, 689, 936

[13] Jurić, M. et al. 2008, ApJ, 673, 864

[14] Kollmeier, J. A. et al. 2009, ApJ, 705, 158 
Assembling the Puzzle of the Milky Way

[15] Sesar, B. et al. 2007, AJ, 134, 2236

[16] Sesar, B. et al. 2010a, ApJ, 708, 717

[17] Sesar, B., Vivas, A. K., Duffau, S. \& Ivezić, Ž. 2010b, ApJ, 717, 133

[18] Sesar, B., Jurić, M. \& Ivezić, Ž. 2011, ApJ, 731, 4

[19] Watkins, L. L. et al. 2009, MNRAS, 398, 1757

[20] York, D. G. et al. 2000, AJ, 120, 1579 